\title{
DEM simulation of the granular Maxwell's Demon under zero gravity
}

\author{
Wenguang Wang ${ }^{1,2}$, Zhigang Zhou ${ }^{1,2}$, Jin Zong ${ }^{1}$, and Meiying Hou ${ }^{1,2, *}$ \\ ${ }^{1}$ Key Laboratory of Soft Matter Physics, Beijing National Laboratory for Condense Matter Physics, Institute of Physics, Chinese \\ Academy of Sciences, 100190 Beijing, China \\ ${ }^{2}$ School of Physical Sciences, University of Chinese Academic of Sciences, 100049 Beijing, China
}

\begin{abstract}
In this work, granular segregation in a two-compartment cell (Maxwell's Demon) under zero gravity is studied numerically by DEM simulation for comparison with the experimental observation in satellite SJ-10. The effect of three parameters: the total number of particles $N$, the excitation strength $\Gamma$, and the position of the window coupling the two compartments, on the segregation ${ }^{\varepsilon}$ and the waiting time $\tau$ are investigated. In the simulation, non-zero segregation under zero gravity is obtained, and the segregation $\varepsilon$ is found independent of the excitation strength $\Gamma$. The waiting time $\tau$, however, depends strongly on $\Gamma$. For higher acceleration $\Gamma,{ }^{\mid}{ }_{i} \mid$ reaches steady state value ${ }^{\varepsilon}$ faster.
\end{abstract}

\section{Introduction}

Granular materials' extremely rich dynamical behaviours have attracted attentions of physicists of different fields in recent years[1-3]. Examples are the heap formation of a granular bed[4-6] and the size segregation of a granular system with grains of various sizes under vertical vibration known as the Brazil Nut effects[7,8]. In these phenomena, energy is being injected continuously into the system by the oscillating boundaries and propagated into the bulk by the inelastic collisions of the grains. A steady state of the whole system is reached when the dissipation of the system is balanced by the input of the energy. Since both the energy input and dissipation depends crucially on the configurations of the system, many intriguing steady states, such as granular segregation in a twocompartment cell known as Maxwell's Demon phenomenon $[9,10]$ and even oscillatory states can be observed. The segregation phenomenon relies on the existence of a non-monotonic flux, which determines the number of particles per unit time flows between the two compartments[11]. The flux function is derived from the equation of gas state and therefore depends on the gravity. If applicable, the phenomenon can be used to transport granular materials in space[12,13]. In this work, we therefore perform simulation study to find the condition for possible segregation in two-compartment granular gas system in an environment of zero gravity. We find that (1) density gradient exists in the cell along excitation direction that particles gather near the end away from the vibration wall; (2) segregation among the two compartments exists even for very small excitation acceleration $\Gamma$ as long as number of particles $N$ exceeds a critical number and the waiting time is long enough;
(3) different from the situation in gravity, segregation $\varepsilon$ is independent of $\Gamma$ when in zero gravity; (4) the waiting time $\tau$ is shorter when the acceleration $\Gamma$ is higher, but reaches a minimum when $\Gamma$ is large enough.

\section{Model}

The simulation is based on discrete element method, in which each particle is treated as a discrete element[14]. The motion of each particle obeys the Newton's Second Law. The interaction between them is considered if and only if two particles collide. Taking the rotation into consideration, the particles' equations of motion are as follows:

$$
\begin{gathered}
m_{i} \ddot{\vec{r}}_{i}=\sum_{j, j \neq i} \vec{F}_{i j}^{C}+\vec{F}_{i}^{O}, \\
I_{i} \dot{\vec{\omega}}_{i}=\sum_{j, j \neq i} \vec{R}_{i} \times \vec{F}_{i j}^{C}+\vec{M}_{i}^{O},
\end{gathered}
$$

where $^{m_{i}}, I_{i}, \vec{r}_{i}$ and $\vec{\omega}_{i}$ are the mass, the moment of inertia, the displacement ${ }_{F}$ and the angular velocity of particle $i$, respectively. $F_{i j}^{C}$ is the contact force that the particle $i$ is applied by the particle ${ }^{j} \cdot \vec{R}_{i}$ is the radius vector of particle $i \cdot \vec{F}_{i}^{O}$ and $\vec{M}_{i}^{O}$ are other forces and torques applied to the particle ${ }^{i}$, such as the gravity force. Generally, $F_{i j}^{C}$ can be decomposed into two parts: a normal component and a tangential one,

$$
\vec{F}_{i j}^{C}=\vec{F}_{i j}^{n}+\vec{F}_{i j}^{t} \text {. }
$$

The normal and the tangential contact forces between two particles are handled separately.

\footnotetext{
* Corresponding author: mayhou@iphy.ac.cn
} 
Two particles interact with each other only during contact. The overlap in the normal direction between particles $i$ and ${ }^{j}$ with radii $R_{i}$ and $R_{j}$, respectively, is

$$
\delta=\left(R_{i}+R_{j}\right)-\left(\vec{r}_{i}-\vec{r}_{j}\right) \cdot \vec{n},
$$

Where $\delta$ is a positive number when the two particles interact, $\vec{n}$ is the unit vector of the relative displacement $\vec{n}=\left(\vec{r}_{i}-\vec{r}_{j}\right) /\left|\vec{r}_{i}-\vec{r}_{j}\right|$. Considering the dissipative effect, the equation of the normal contact force is

$$
F_{i j}^{n}=k_{n} \delta+\gamma_{n} v_{n}
$$

where $k_{n}$ and $\gamma_{n}$ are the spring stiffness and the dissipative coefficient, respectively, and $\vec{v}_{n}$ is the normal component of the relative velocity $\vec{v}_{i j}=\vec{v}_{i}-\vec{v}_{j}$. This is called Linear Spring Dashpot (LSD) model.

The tangential contact force is determined by the tangential component of the relative velocity and the normal contact force

$$
F_{i j}^{t}=\min \left(\gamma_{t} v_{i j}^{t}, \mu F_{i j}^{n}\right),
$$

Where $\gamma_{t}$ is a numerical constant, $\mu$ is the friction coefficient and $v_{i j}^{t}$ is the tangential component of $\vec{v}_{i j}$. Fig. 1 shows the mechanism of the contact model in this work.

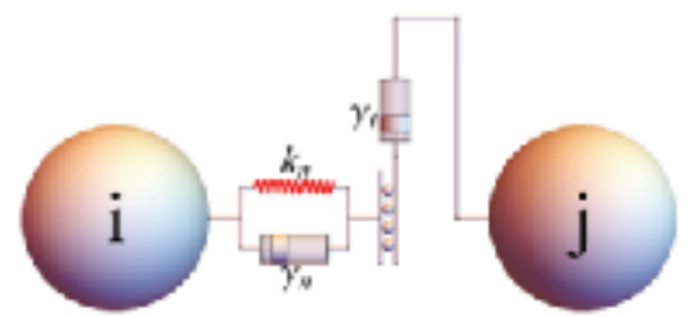

Fig. 1. The mechanism of the contact model.

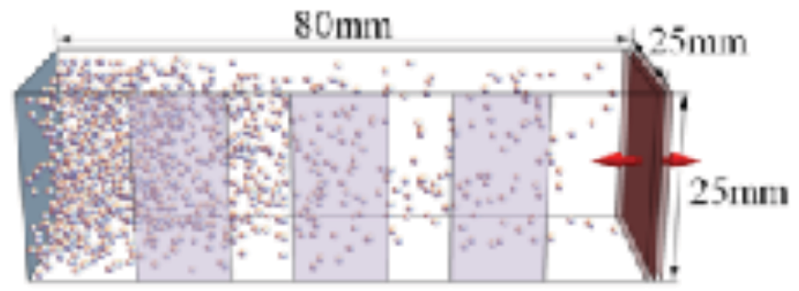

Fig. 2. Schematic of the virtual-window single cell. Particles are excited by the right-end moving wall. The virtual window at three different locations are indicated by rectangular shades at the side wall.

The numerical parameters of the granular cell system are taken same as that in the experiments. In the simulation, the particle radius $R$ is $0.5 \mathrm{~mm}$. The density $\rho$ of particles is $4500 \mathrm{~kg} / \mathrm{m}^{3}$. The coefficient of restitution $e$ for grain-grain collision is taken as 0.8 . The coefficient of frication ${ }^{\mu}$ is 0.3 . The total number of particles in the connected two-compartment cell is taken as $N$. Let the number in cell one (say upper one) be $N_{1}$, the number in the other (lower) cell is then $N_{2}=N-N_{1}$. To start with, we put particles evenly in each compartment, $N_{1}=N_{2}=N / 2$. The initial velocity is arbitrarily set to be $0.1 \mathrm{~m} / \mathrm{s}$ with random direction.

In order to find a flux function for the segregation condition in microgravity, i.e., to know the number of particles flow instantaneously through the window per unit time, we count the number of particle-wall collisions at a virtual window of a single cell[9] shown in Fig. 3. The size of the single-cell is $80 \mathrm{~mm} \times 25 \mathrm{~mm} \times 25 \mathrm{~mm}$. Three rectangular shades along the side wall are shown in Fig. 3 to indicate the virtual windows at different positions. We record how many times particles collide with a given virtual window at a time interval for a sufficiently long time. The flux profile can then be found by the counts of collisions per unit time across a given virtual window.

\section{Results}

\subsection{Flux function obtained in virtual-window single-cell simulation}

Finding flux function is helpful in modelling the occurrence of the segregation. The segregation is governed by the following equation:

$$
\frac{d N_{i}(t)}{d t}=-F\left(N_{i}(t)\right)+F\left(N-N_{i}(t)\right), \quad i=1,2,
$$

where the $N_{1}(t)$ and $N_{2}(t)$ are the counts of particles in the two compartments at time t, and $F\left(N_{i}\right)_{\text {is }}$ the number of particles per unit time at time $t$ that flow from a compartment containing $N_{i}$ particles to another compartment. Once knowing the function $F\left(N_{i}\right)$, $N_{i}(t)$ can be obtained from Eq. (10).

In order to characterize the segregation of particles in the two cells, a dimensionless parameter $\varepsilon_{i}$ is introduced:

$$
\varepsilon_{i}=\left(N_{i}-N / 2\right) / N, \quad i=1,2 .
$$




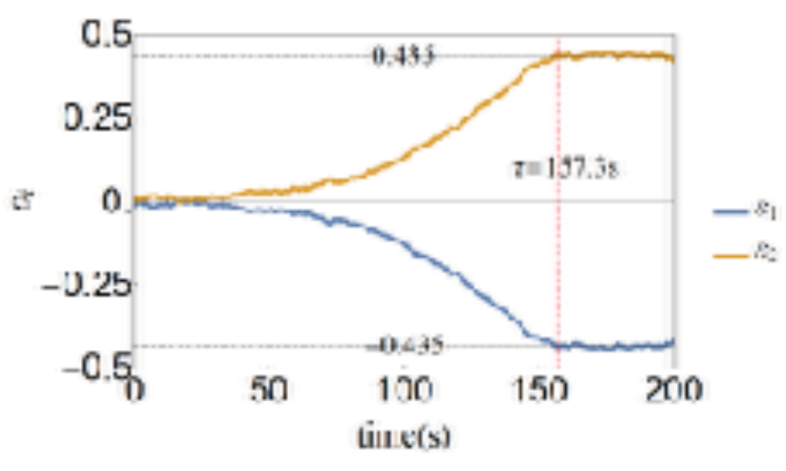

Fig. 3. One example of simulation result of $\varepsilon_{i}(t)$

Fig. 3 shows a plot of simulation result how $\varepsilon_{i}$ changes with time. It takes some time for the population $N_{i}(t)$ to reach a steady state. We call this time as the waiting time $\tau$. At any time $\varepsilon_{1}(t)=-\varepsilon_{2}(t)$, and the absolute value of ${ }^{\varepsilon_{i}}$ at the steady state is indicated as $\varepsilon$. $\varepsilon=0$ means particles are equally populated in the two cells, and $|\varepsilon|=0.5$ means all the particles gather in one compartment, i.e., fully segregated.

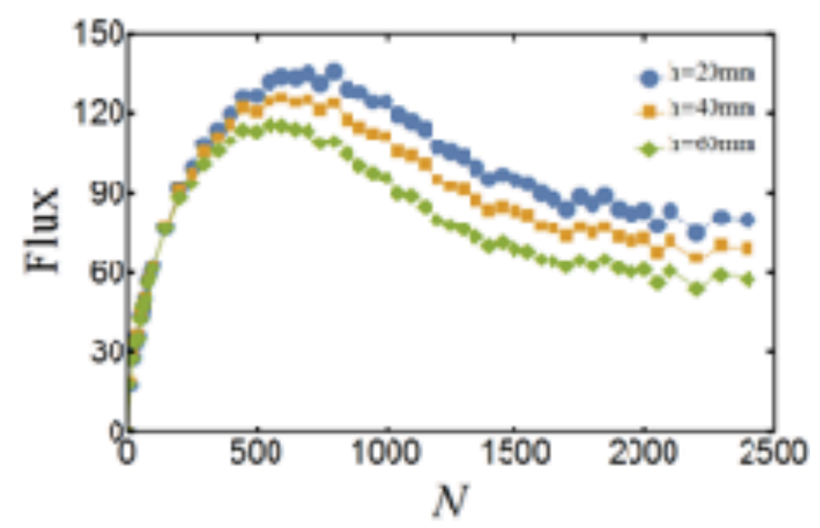

Fig. 4. Flux as a function of the number $N$.

The flux function obtained by the virtual-window singlecell simulation method[9] is shown in Fig. 4 for three locations of the window. A non-monotonic function $F\left(N_{i}\right)$ guaranteed the occurrence of the segregation. A weakly position dependence of the flux function shown in Fig. 4 tells us that flux value is greater when the window position is moving away from the oscillating wall.

\subsection{The effect of total number $N$ on the segregation $\varepsilon$}

Initially particles are distributed equally in the two cells. Segregation appears after about 100 seconds, and is fully developed 200 seconds afterwards.

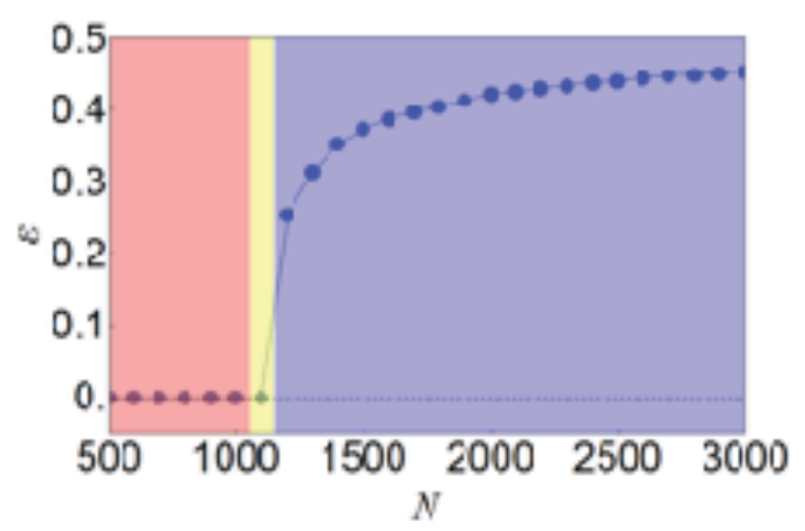

Fig. 5. Variation of the asymmetry parameter ${ }^{\varepsilon}$ in the steady state as a function of $N$.

With the designed setup parameters, Fig. 5 gives us the simulation results how $\varepsilon_{i}(t)$ changes with the total number $N$ from 800 to 1500 . When the total number $N$ is less than or equal to 1100 , the asymmetry ${ }^{\varepsilon}$ fluctuate around 0 . Segregation does not occur. Only when $N$ exceeds a critical value (here the critical value is about $1100)$ the segregation occurs. The asymmetry parameter ${ }^{\varepsilon}$ grows from 0 and approaches 0.5 with increasing $N$.

\subsection{Effect of excitation acceleration on the segregation}

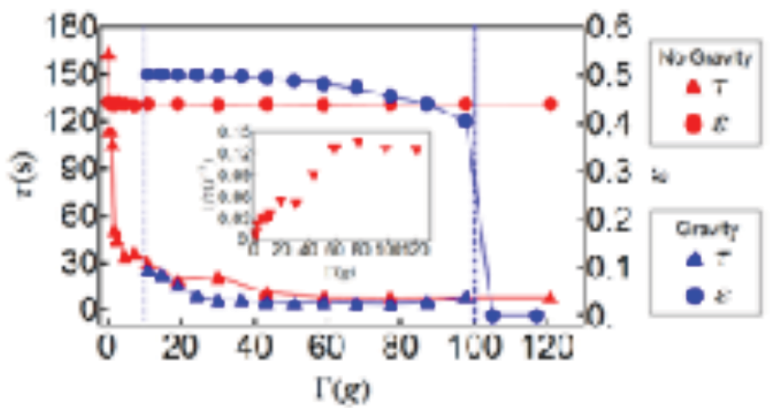

Fig. 6. Effects of the vibration intensity on the asymmetry parameter $\varepsilon$ and the waiting time $\boldsymbol{\tau}$ with and without gravity.

Fig. 6 gives the simulation results of the asymmetry parameter $\left|\varepsilon_{i}\right|$ changing with time under different $\Gamma$. For higher acceleration $\Gamma,\left|\varepsilon_{i}\right|$ reaches steady state value ${ }^{\varepsilon}$ faster.

Since in microgravity experiments the operational time is normally limited, the waiting time ${ }^{\tau}$ is important to know in advance, which tells us how long to wait for seeing the effect. Shown in Fig. 6 , the rate ${ }^{1 / \tau}$ seems linearly proportional to $\Gamma$ at low $\Gamma$. Only when $\Gamma$ is high enough the rate ${ }^{1 / \tau}$ reaches a maximum value. For the $\Gamma$ value available in our microgravity experiment (normally less than 1g), Fig. 6 tells us the time duration $\tau$ shall be of the order of 100 seconds. This result helps us in determining the experimental design. 
In Fig. 6, it shows that the waiting time ${ }^{\tau}$ does not go to zero, there is a minimum time required for particles to go to the steady segregation state no matter how strong the vibration intensity goes. In our case the value is of the order of 10 seconds.

The above numerical results are quite different with the experiments in gravity. With gravity particles act like gas molecules, and distribute in both cells. No segregation of the granular gas is observed at high $\Gamma$ when particle random collisions dominate. When $\Gamma$ is below a certain threshold, no segregation is observed either as the gravitational force dominates. With no gravity, the segregation $\varepsilon$ becomes a constant and does not depend on $\Gamma$, as seen in Fig. 6 , but the waiting time $\tau$ depends strongly on $\Gamma$ with or without gravity.

\section{Conclusion}

Our simulation shows granular segregation is achievable at zero gravity, although the condition can be different from the case under gravity. With gravity, the segregation depends on $\Gamma$ and can be divided into three regimes: one at low $\Gamma$ when the gravitational force is dominant that no segregation appears and ${ }^{\varepsilon}$ is zero; the second regime when $\Gamma$ is at an intermediate value that segregation appears $\varepsilon$ becomes non-zero; the third regime when $\Gamma$ is high enough that random particle collisions dominate and ${ }^{\varepsilon}$ becomes zero again. Under zero gravity, segregation ${ }^{\varepsilon}$ does not depend on $\Gamma$. It is a constant and is determined by the geometry of the cell. A minimum excitation time is necessary to observe the phenomenon with or without gravity. This time $\tau$, however, depends strongly on the value $\Gamma$. With the affordable acceleration $\Gamma$ in satellite condition, the excitation time is on the order of a few minutes, which means no segregation can be seen in drop tower or parabolic experiments[1,15]. Both offer short time, 3 seconds to 22 seconds, microgravity condition.

\section{Acknowledgments}

Project supported by the "Strategic Priority Research Program-SJ-10" of the Chinese Academy of Sciences (Grant No.XDA04020200), and the National Natural Science Foundation of China (Grant Nos. 11474326, 11274354).

\section{References}

1. D. van der Meer, K. van der Weele and D. Lohse, Phys. Rev. E 63061304 (2001)

2. B. Meerson, T. Pōschel, P. V. Sasorov and T.
Schwager, Phys. Rev. E 69021302 (2004)

3. G. Costantini, D. Paolotti, C. Cattubo and U.M.B. Marconi , Physica A 347 411-428 (2005)

4. R. Lambiotte, J.M. Salazar and L. Brenig Phys. Lett. A 343 224-230 (2005)

5. Liu R, Li Y and Hou M, Phys. Rev. E 75 061304(2007)

6. Liu R, Li Y and Hou M, Phys. Rev. E 79 052301(2009)

7. Hou M, Li Y, Liu R, Zhang Y and Lu K, Phys. Status Solidi A 207 2739-2479 (2010)

8. Li Y, Hou M and P. Evesque, J. Phys. Conf. Ser. 327 012034(2011)

9. Li Y, Liu R, M. Shinde and Hou M, Granular Matter 14 137-143(2012)

10. Li Y, Liu R and Hou M, Phys. Rev. Lett. 109 198001(2012)

11. J. Eggers, Phys. Rev. Lett. 83 5322-5325(1999)

12. E. Opsomer, M. Noirhomme, N. Vandewalle and F. Ludewig, Phys. Rev. E 88 012202(2013)

13. M. Noirhomme, E. Opsomer, N. Vandewalle and F. Ludewig, Eur. Phys. J. E 38(2015)

14. S. Luding, Granular Matter 10 235-246(2008)

15. Wang H, Chen Q, Wang W G and Hou M Y, Acta. Phys. Sin. 65 014502(2016) 